\title{
Successful Trial Design and Planning in Systemic Sclerosis: Does It Take a Village?
}

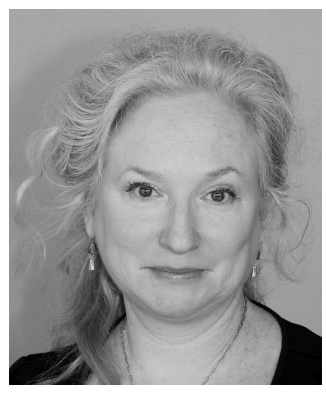

Negative trial reporting, especially in rare diseases, provides essential information. The design and analysis of a "negative" trial, in diseases for which few potential treatments and no cure exist, warrants particular scrutiny to determine whether the trial was truly negative, or rather a "failed" trial owing to other design, financial, planning, or recruitment impediments ${ }^{1}$. A trial that is discontinued early warrants the label negative if an unbiased, independent data safety monitoring board identified either a harm signal (excessive intolerance or drug-related adverse events) or a clear worsening of the disease state in comparison to placebo.

In an article by Hsu, et al, in this issue of The Journal, the authors describe a 52-week randomized double-blind placebo-controlled multicenter phase II study of pomalidomide $^{2}$. The sponsors have conceded to the reviewers that the study was discontinued early because of poor recruitment. Yet the article's discussion section persists in stating that discontinuation by the sponsor was due to lack of drug efficacy. To be clear, there exists no statistical application capable of calculating a reliable result in any of the study variables between the 4 treatment and the 7 placebo cases completing a study that was powered for a sample of 88 . Thus, the study results carry similar statistical weight to the initial anecdotal case reports of perceived efficacy of pomalidomide by systemic sclerosis ( $\mathrm{SSc}$ ) specialists cases that likely inspired moving the drug to trial.

SSc disease behavior remains complex and challenging to the most seasoned SSc clinician researchers who are well-acclimated to a cautious approach to data interpretation in this "rare" and exceptionally complex disease. In a journal less cognizant of the humbling landscape in SSc clinical trial design, this study would have slipped by as a negative study, generating an inaccurate interpretation of the conclusions in the less discriminant reader, who could have been an insurance provider or a grant reviewer presiding over a decision to grant compassionate treatment or to fund a new study in this drug class. Though declined as negative, the study is rightly afforded publication as an occasion to pause and collectively consider the complexity of design, preparation, and planning for a trial in SSc.

When patients with life-threatening progressive conditions such as certain malignancies or SSc enroll in clinical trials, patient life-years are bartered, in good faith, against the likelihood that a trial will at least provide information beneficial to future patients and researchers even if it does not have self-benefit. Patients bravely provide consent against the possibility of irreversible disease progression and death, potentially sacrificing otherwise currently acceptable treatment strategies, as well as forgoing participation in other (perhaps better designed) available trials.

Further, each clinical trial ties up the most premium research resource: patients. When multiple trials recruit concurrently, it potentially impedes enrollment across the SSc research community, thereby protracting study time frames, increasing costs, and financially dissolving trials unprepared for this. Such a scenario is equally applicable in trials in other rare diseases. Therefore, exquisite care and clarity in study design, preparation, planning, and implementation are required to safeguard investment of patient life-years and the provision of valuable insight into treatment efficacy and safety, and perhaps even disease behavior and subtyping. In this trial, the demands of continuing a trial with poor chances of efficiently producing meaningful results could not be met. Rather than embroiling patients and finances further, an appropriate decision was made to halt recruitment.

SSc research is fraught with idiosyncrasies related to disease and outcome measures. For example, we are learning that, depending on the targeted manifestation, SSc may demand longer study duration, to allow for improved demonstration of treatment response, than for example in idiopathic pulmonary arterial hypertension, with SSc lung studies extending treatment by 6 to 12 months $^{3}$.

The single most enigmatic challenge to clinical trial design in progressive, potentially obliterative multisystem autoimmune diseases such as SSc, vasculitis, and sarcoidosis

See Pomalidomide with ILD in SSc, page 405

Personal non-commercial use only. The Journal of Rheumatology Copyright (C) 2018. All rights reserved. 
is deciphering irreversible, untreatable damage from active, progressive, treatment-responsive disease. Prolonged disease duration, especially in SSc-interstitial lung disease (ILD), is associated with a higher likelihood that less treatment-responsive damage exists and improvement is increasingly less discernible in a clinical trial. Trials are optimally designed to select for the likelihood of a subject to respond if exposed to a treatment that is effective - this is called "cohort enrichment." The stretching of disease duration out to 7 years ${ }^{4}$ seems safer relegated to trials testing safety rather than efficacy ${ }^{5}$. Though SSc-ILD can continue to progress after 5 years, the greatest volume loss occurs in the first 2 years of onset, followed by much smaller increments thereafter ${ }^{6,7}$. Therefore, limiting disease duration until a more discriminating disease activity detection algorithm (which may depend upon disease subtype or target organ) can be identified is a vital consideration to trial criteria. Comparative disease duration is also essential descriptive information between trial arms.

In this trial, the authors speculate that cohort enrichment strategies were overly restrictive and resulted in poor enrollment. However, the study criteria do not differ greatly from other concurrent SSc-ILD trials successfully meeting recruitment ${ }^{5,8}$, except for variance regarding permissiveness of concurrent therapies. The reinforcement criteria for a baseline forced vital capacity (FVC) 70-80\%, requiring demonstration of $\mathrm{a} \geq 5 \%$ drop within a prior 2 -year period, is a good enhancement, albeit still liberal in a stratum in which progressive disease is expected to decline by large increments ${ }^{4,5}$. Importantly, the vagueness and lack of standardization surrounding inclusion of pulmonary hypertension $(\mathrm{PH})$ is curious, a vital distinction to preserving cohort enrichment. Here, the simple application of FVC:DLCO ratio ${ }^{9,10,11}$, designed to aid in distinction of ILD versus $\mathrm{PH}$ predominance, might have enhanced cohort enrichment.

The modified Rodnan skin score (mRSS), as a primary outcome measure, is often used in clinical trials, yet only ever validated in early diffuse disease (about initial 24 mos). Its limitations and enrichment potential over time have been described $^{12}$. While the mRSS and ILD correlate well and make sense as co-primary endpoints, the University of California at Los Angeles Scleroderma Clinical Trial Consortium Gastrointestinal Tract (SCTC-GIT), a lengthy questionnaire addressing gastrointestinal symptomatology, lacks clarity in this trial as a primary endpoint choice. Here, the SCTC-GIT displaces well-validated relevant patient-reported instruments in an ILD trial, such as those measuring respiratory symptoms as well as the Scleroderma Health Assessment Questionnaire, a widely used, strongly validated, user-friendly outcome measure incorporating several additional validated scales that assess central SSc manifestations potentially responsive to treatment, including a validated lung symptom scale. These measures were relegated to secondary and "exploratory" assessments - and in the absence of robustly validated health-related quality of life (HRQoL) instruments such as the patient's global assessment of disease activity, the Medical Outcomes Study Short Form-36, or EQ-5D. HRQoL is a parallel competitor to survival, as perceived by those living with pulmonary fibrosis ${ }^{13,14}$.

Concurrent SSc and SSc-ILD trials appear to have met recruitment targets ${ }^{5,8,15}$. There are 56 enrollment sites listed on clinicaltrials gov for this trial ${ }^{16}$. Did site investigators lose confidence in the drug, study design, or financial feasibility? Or perhaps concurrent trials were competing at single sites with other trials that were more appealing or convincing. Was there hesitation to enroll sick patients in a placebo-controlled study without permissive background or rescue therapy or the reassurance of frequent FVC assessments to identify and rescue declining health sooner?

Since the demonstration of delayed ILD progression with cyclophosphamide in the SLS I trial ${ }^{17}$, controversy persists surrounding the ethics and science of placebo-only versus allowance of background therapy with placebo. This study seems likely to have accommodated "niche" patients - those intolerant of, failing, or unable to attain other available therapies or trial enrollment; compounded by investigator recruiting tendencies/fears expressing the weight of community sentiment regarding placebo, thus impairing recruitment numbers.

Assuring accountability, accuracy, and clarity in treatment-data reporting is crucial in a disease of scarce therapeutic options. This drug class with promising preclinical and clinical treatment properties has not been appropriately evaluated in this disease, and to discard its future use and research would be, at this time, a disservice.

We are heavily reliant upon partnership with industry to persevere in addressing treatment gaps in SSc. This partnership needs to be fostered with transparency and a strengthening of shared knowledge and patient-centered priorities. The reporting of this study's results is somewhat confusing, requiring significant effort to establish actuality between the data tables and the narrative, with initial manuscript submissions mistaking the actual conclusion. As trial numbers in SSc continue to escalate, to assure the best use of resources and trial outcomes, the SSc research community needs to contemplate a pre- and post-study endorsement mechanism sanctioned by a global knowledgeable equanimous community, such as the SCTC.

This could involve a trial design preview for crucial preparatory guidance before a study is initiated, whereby the nuts and bolts of study design could be reviewed as well as alerting potential trialists to design enhancements and possible community sensibilities such as background/rescue therapy that could impair recruitment, and importantly, a poststudy review of results and interpretation. The poststudy endorsement process might include a template-based appli-

Personal non-commercial use only. The Journal of Rheumatology Copyright @ 2018. All rights reserved. 
cation/examination of a composite containing the study protocol, an analytic results questionnaire, a summarized interpretation of the data (free of narrative distraction), and a succinct addendum to qualify any potential irregularities or impediments in protocol or data acquisition. It is true that a risk of delayed reporting exists with such a system, but the benefits to research, use of resources, and patient care are a worthy exchange. Such an endorsement body could tandemly protect and promote much-needed creative examination of enrichment and design enhancements.

Lastly, in clinical trial design, the input of an expert is often overlooked and inherently important. This expert is the patient. Patient advisers/research partners intimately understand the logistics of living with SSc and can provide great insight on feasibility, compliance, and concerns about clinical trial design.

\section{LESLEY ANN SAKETKOO, MD, MPH,}

Tulane University School of Medicine, New Orleans Scleroderma and Sarcoidosis Patient Care and Research Center, University Medical Center Comprehensive Pulmonary Hypertension Center, New Orleans, Louisiana, USA.

Address correspondence to Dr. L.A. Saketkoo, MD, MPH, Associate Professor of Medicine, Tulane University School of Medicine, Division of Pulmonary Medicine and Critical Care, 1430 Tulane Ave., New Orleans, Louisiana 70112,USA.E-mail: 1saketk@tulane.edu

\section{REFERENCES}

1. Goldacre B. How to get all trials reported: audit, better data, and individual accountability. PLoS Med 2015;12:e1001821.

2. Hsu VM, Denton CP, Domsic RT, Furst DE, Rischmueller M, Stanislav M, et al. Pomalidomide in patients with interstitial lung disease due to systemic sclerosis: a phase II, multicenter, randomized, double-blind, placebo-controlled, parallel-group study. J Rheumatol 2018;45:405-10

3. Galiè N, Olschewski H, Oudiz RJ, Torres F, Frost A, Ghofrani HA, et al. Ambrisentan for the treatment of pulmonary arterial hypertension: results of the Ambrisentan in Pulmonary Arterial Hypertension, Randomized, Double-Blind, Placebo-Controlled, Multicenter, Efficacy (ARIES) study 1 and 2. Circulation. 2008;117:3010-19.

4. Khanna D, Tseng CH, Farmani N, Steen V, Furst DE, Clements PJ, et al. Clinical course of lung physiology in patients with scleroderma and interstitial lung disease: analysis of the Scleroderma Lung Study Placebo Group. Arthritis Rheum 2011; 63:3078-85.
5. Khanna D, Albera C, Fischer A, Khalidi N, Raghu G, Chung L, et al. An open-label, phase II study of the safety and tolerability of pirfenidone in patients with scleroderma-associated interstitial lung disease: the LOTUSS trial. J Rheumatol 2016;43:1672-9.

6. Steen VD, Lanz JK Jr, Conte C, Owens GR, Medsger TA Jr. Therapy for severe interstitial lung disease in systemic sclerosis. A retrospective study. Arthritis Rheum 1994;37:1290-6.

7. Steen VD, Medsger TA Jr. Severe organ involvement in systemic sclerosis with diffuse scleroderma. Arthritis Rheum 2000; 43:2437-44.

8. Khanna D, Denton CP, Jahreis A, van Laar JM, Frech TM, Anderson $\mathrm{ME}$, et al. Safety and efficacy of subcutaneous tocilizumab in adults with systemic sclerosis (faSScinate): a phase 2, randomised, controlled trial. Lancet 2016;387:2630-40.

9. Steen VD, Graham G, Conte C, Owens G, Medsger TA Jr. Isolated diffusing capacity reduction in systemic sclerosis. Arthritis Rheum 1992;35:765-70.

10. Steen V, Medsger TA Jr. Predictors of isolated pulmonary hypertension in patients with systemic sclerosis and limited cutaneous involvement. Arthritis Rheum 2003;48:516-22.

11. Chung L, Liu J, Parsons L, Hassoun PM, McGoon M, Badesch DB, et al. Characterization of connective tissue disease-associated pulmonary arterial hypertension from REVEAL: identifying systemic sclerosis as a unique phenotype. Chest 2010;138:1383-94

12. Dobrota R, Maurer B, Graf N, Jordan S, Mihai C, Kowal-Bielecka $\mathrm{O}$, et al; EUSTAR coauthors. Prediction of improvement in skin fibrosis in diffuse cutaneous systemic sclerosis: a EUSTAR analysis. Ann Rheum Dis 2016;75:1743-8.

13. Russell AM, Scholand M, Snyder EA, Russell A, Burdett C, Doyle AM, et al. Impact, survival, symptoms and management: US and UK patient perceptions of living with idiopathic pulmonary fibrosis, international conference of the American Thoracic Society (ATS). New York: American Thoracic Society; 2016:1073-449.

14. Saketkoo LA, Mittoo S, Huscher D, Khanna D, Dellaripa PF, Distler $\mathrm{O}$, et al; CTD-ILD Special Interest Group. Connective tissue disease related interstitial lung diseases and idiopathic pulmonary fibrosis: provisional core sets of domains and instruments for use in clinical trials. Thorax 2014;69:428-36.

15. Tashkin DP, Roth MD, Clements PJ, Furst DE, Khanna D, Kleerup EC, et al; Scleroderma Lung Study II Investigators. Mycophenolate mofetil versus oral cyclophosphamide in scleroderma-related interstitial lung disease (SLS II): a randomised controlled, double-blind, parallel group trial. Lancet Respir Med 2016; 4:708-19.

16. Trial enrollment sites. [Internet. Accessed November 11, 2017.] Available from: clinicaltrials.gov/ct2/show/NCT01559129

17. Tashkin DP, Elashoff R, Clements PJ, Goldin J, Roth MD, Furst DE, et al; Scleroderma Lung Study Research Group. Cyclophosphamide versus placebo in scleroderma lung disease. N Engl J Med 2006;354:2655-66.

J Rheumatol 2018;45:297-9; doi:10.3899/jrheum.180062 